\title{
DESENVOLVIMENTO DE UM PROTOCOLO OPERACIONAL PADRÃO PARA ENFERMEIROS NO CUIDADO A CRIANÇAS VÍTIMAS DE VIOLÊNCIA
}

\section{DEVELOPMENT OF A STANDARD OPERATIONAL PROTOCOL FOR NURSES IN THE CARE OF CHILDREN VICTIMS OF VIOLENCE}

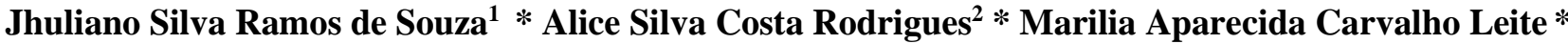 \\ Camila Mendonça de Moraes ${ }^{3} *$ Patrícia Scotini Freitas $^{4} *$ Namie Okino Sawada $^{5}$
}

\begin{abstract}
RESUMO
Objetivo: elaborar um protocolo de procedimento operacional padronizado referente ao atendimento de enfermeiros da estratégia saúde da família no cuidado às crianças vítimas de violência. Método: estudo metodológico, seguindo o modelo do clico de ação dividido nas etapas: I) Identificação das lacunas do conhecimento; 2) Adaptação do conhecimento ao contexto local; 3) Avaliação das barreiras para uso do conhecimento; 4) Seleção, adaptação e implementação das intervenções; 5) Monitoramento do uso do conhecimento; 6) Avaliação dos resultados e 7) Uso sustentável do conhecimento. Resultados: Construiu-se um fluxograma que abordassem as etapas necessárias para a assistência à criança vítima de violência infantil por meio da consulta de enfermagem, notificação compulsória, comunicação ao Conselho Tutelar e o encaminhamento aos serviços especializados. Conclusão: estudo desenvolveu um protocolo operacional padrão com o intuito de padronizar a ação referente ao atendimento de enfermeiros da estratégia saúde da família no cuidado a crianças vítimas de violência.
\end{abstract}

Palavras-chave: Processo de Enfermagem; Maus-Tratos Infantis; Estratégia Saúde da Família; Cuidados de Enfermagem; Prática Baseada em Evidencias; Translação do conhecimento.

\begin{abstract}
Objective: to develop a standardized operating procedure protocol for the care of nurses in the family health strategy in caring for children who are victims of violence. Method: methodological study, following the action cycle model divided into steps: I) Identification of knowledge gaps; 2) Adaptation of knowledge to the local context; 3) Assessment of barriers to the use of knowledge; 4) Selection, adaptation and implementation of interventions; 5) Monitoring the use of knowledge; 6) Evaluation of results and 7) Sustainable use of knowledge. Results: A flowchart was built that addressed the necessary steps to care for a child victim of child violence through nursing consultation, compulsory notification, communication to the Guardianship Council and referral to specialized services. Conclusion: a study developed a standard operational protocol in order to standardize the action related to the care of nurses in the family health strategy in caring for children who are victims of violence.

Keywords: Nursing Process; Child Abuse; Family Health Strategy; Nursing Care; Evidence-Based Practice; Knowledge Translation.
\end{abstract}

\footnotetext{
1 Enfermeiro. Doutorando em Enfermagem. Programa de Pós-Graduação em Enfermagem. Alfenas (MG), Brasil. ORCID: http://orcid.org/0000-0002-4338-4433. E-mail: jhulianoramoz@ hotmail.com

2 Enfermeira. Doutoranda em Enfermagem. Programa de Pós-Graduação em Enfermagem. Alfenas (MG), Brasil. ORCID: https://orcid.org/0000-001-7788-3989 E-mail: alicescosta14@gmail.com ORCID: https://orcid.org/0000-0003-1438-6453 E-mail: lyla.leite@hotmail.com

${ }^{3}$ Enfermeira. Doutora em Ciências. Professora Adjunta da Universidade Federal do Rio de Janeiro. Macaé (RJ), Brasil. ORCID: https://orcid.org/0000-0001-8954-7616 E-mail: camilamendonca1@ hotmail.com

${ }^{4}$ Doutora em Ciências pela EERP-USP, na área de Enfermagem Fundamental CAPES 7 (2014). ORCID: https://orcid.org/00000002-7469-6034 E-mail: patricia.freitas@unifal-mg.edu.br

${ }^{5}$ Doutorado em Enfermagem. ORCID: https://orcid.org/0000-0002-1874-3481 E-mail: sawada@eerp.usp.br
} 


\section{INTRODUÇÃO}

O termo sobre violência é definido pela Organização Mundial de Saúde como "toda forma de maus-tratos emocionais e/ou físicos, abuso/ou exploração sexual, negligência ou danos potenciais ou reais à saúde das crianças" (1).

No Brasil, existem legislações que visam a proteção de crianças e adolescentes contra a violência, como o Estatuto da Criança e do Adolescente (ECA) e a Lei Menino Bernardo, tendo objetivos em comum como o fortalecimento ao combate contra crimes de pedofilia, investigação de crimes cibernéticos contra a dignidade sexual, instituindo um padrão de políticas direcionadas ao combate à violência, com zelo aos direitos humanos ${ }^{(2-3-4)}$.

No entanto, ainda existem subnotificações sobre casos de violência infantil nos serviços de saúde, pelo medo da vítima ao denunciar o perpetrador e as falhas no atendimento. Além disso, crianças e adolescentes são públicos vulneráveis por não conseguirem se defender e ter voz para reportar sobre esse tipo de crime, os colocando em um dos principais grupos que sofrem violência ${ }^{(5)}$.

No contexto assistencial, destaca-se o atendimento de crianças vítimas de violência na Estratégia Saúde da Família (ESF), sendo um serviço público que beneficia a identificação, o acolhimento, a notificação e o encaminhamento aos setores especializados, porém, ainda existem falhas no atendimento pelos profissionais de enfermagem e os meios de direcionar e encaminhar as vítimas, de modo a não revitimizá-las, demonstrando falta de treinamento pelos mesmos nos cuidados prestados ${ }^{(6)}$.

A importância deste estudo concerne na elaboração de um Protocolo Operacional Padrão (POP) que irá auxiliar enfermeiros que atuam na ESF na tomada de decisões frente aos casos de violência infantil na prática clínica, a fim de prestar um cuidado qualificado e justificando-se a relevância desta pesquisa, bem como outras pautadas nesta temática. Diante do exposto, este trabalho tem por objetivo elaborar um POP referente ao atendimento de enfermeiros da ESF no cuidado às crianças vítimas de violência.

\section{MÉTODO}

Trata-se de um estudo metodológico. Esse tipo de estudo envolve investigação dos métodos de obtenção e organização de dados e condução de pesquisas rigorosas, além do desenvolvimento, da validação e da avaliação de ferramentas e métodos de pesquisa. Atualmente crescem as demandas por avaliações de resultados válidos e confiáveis, procedimentos com testes de intervenções rigorosos além de procedimentos sofisticados para a coleta de dados ${ }^{(7)}$. 
Foi adotado o referencial teórico de Graham e colaboradores ${ }^{(8)}$ que traz o modelo do clico de ação, sendo a parte central da investigação do conhecimento por meio de produto gerado pelos pesquisadores, indo a caminho desde a etapas percorridas pela revisão integrativa até os resultados esperados e/ou obtidos por meio da elaboração de um protocolo clínico que irá translacionar o impacto na prática clínica ${ }^{(8-9-10)}$.

Para a elaboração do POP, foi seguido as sete etapas do referencial metodológoco: ${ }^{(8)}$ I) Identificação das lacunas do conhecimento; 2) Adaptação do conhecimento ao contexto local; 3) Avaliação das barreiras para uso do conhecimento; 4) Seleção, adaptação e implementação das intervenções; 5) Monitoramento do uso do conhecimento; 6) Avaliação dos resultados e 7) Uso sustentável do conhecimento.

O presente estudo foi realizado por meio da revisão integrativa da literatura sobre o atendimento de enfermeiros da ESF no cuidado às crianças vítimas de violência, seguindo as etapas: I) identificação do tema e seleção da hipótese; II) estabelecimento de critérios para inclusão e exclusão de estudos; III) definição das informações a serem extraídas dos estudos selecionados; IV) avaliação dos estudos incluídos; V) $\begin{array}{llll}\text { interpretação dos } & \text { resultados } & \text { e } & \text { VI) }\end{array}$ apresentação da revisão/síntese do conhecimento $^{(11)}$.
A coleta de dados foi realizada pelas bases/portais eletrônicos, por meio da Coordenação de Aperfeiçoamento de Pessoal de Nível Superior (CAPES) e da Biblioteca Virtual em Saúde (BVS): Web of Science (WOS); Embase (Elsevier); Literatura LatinoAmericana e do Caribe em Ciências da Saúde (LILACS); Cumulative Index to Nursing and Allied Health Literature (CINAHL) e Medical Literature and Retrivial Sistem onLine (MEDLINE).

A análise dos estudos encontrados se deu por meio da coleta das seguintes variáveis: definição do atendimento de enfermeiros da ESF no cuidado às crianças vítimas de violência, bem como na consulta de enfermagem, anamnese, exame físico, ficha de notificação compulsória e o encaminhamento aos serviços especializados e aspectos ético-legais.

\section{RESULTADOS E DISCUSSÃO}

Por meio do ciclo de ação, foi possível desenvolver uma revisão integrativa da literatura que norteou a confecção do POP para a translação do conhecimento aplicandoo a prática clínica ${ }^{(8-9)}$.

Em vista disso, a translação do conhecimento visa melhorar a qualidade da saúde das pessoas por meio da produção de resultados que possam impactar positivamente na vida do ser humano, bem como na sociedade e nas práticas 
profissionais $^{(10)}$. Desta forma, os pesquisadores delinearam o estudo pelo referencial utilizado seguindo as seguintes etapas:

Na primeira etapa sobre identificação das lacunas do conhecimento para a ação, encontrou-se na literatura científica o déficit de conhecimento da prática profissional em casos de violência infantil, o despreparo e desproteção com relação a tomada de decisão frente aos casos de violência e as dificuldades enfrentadas mediante ao encaminhamento de casos de violência. Além disso, evidenciou-se a escassez de estudos voltados na atuação assistencial dos enfermeiros às crianças vítimas de violência na atenção básica ${ }^{(12)}$.

A translação do conhecimento na prática clínica profissional ${ }^{(8)}$, é importante identificar quais são os stakeholders, sendo pessoas ou organizações que tem interesse na gestão de um projeto ou empresa, podendo ser influenciados de forma positiva ou negativa pelas decisões da empresa/instituição. Dessa forma, a escolha dos stakeholders foram: Enfermeiros (profissionais); Crianças (paciente) e ESF (Instituição).

$\mathrm{Na}$ segunda etapa sobre adaptação do conhecimento ao contexto local, mediante os achados, os pesquisadores propuseram a construção de um POP para nortear os enfermeiros da ESF no atendimento às crianças vítimas de violência. Conforme Quadro 1 descrito na sequência.

Quadro 1 - Procedimento Operacional Padrão (POP), Alfenas, MG, Brasil, 2021

1. NOME:

Atendimento de enfermeiros da Estratégia Saúde da Família no cuidado às crianças vítimas de violência.

\section{PÚBLICO-ALVO:}

Profissionais: Enfermeiros.

Pacientes: Crianças.

Instituição: Estratégia Saúde da Família.

\section{FINALIDADE:}

Garantir um atendimento de qualidade no atendimento de enfermeiros da Estratégia Saúde da Família no cuidado às crianças vítimas de violência. 
4. PROCEDIMENTOS:

1. Identificar os sinais clínicos da violência contra a criança na consulta de enfermagem;

2. Identificar por meio do exame físico as características da violência conta a criança;

3. Notificar por meio da ficha do Sistema de Informação de Agravos a suspeita da violência;

4. Comunicar ao Conselho Tutelar;

5. Realizar o Boletim de Ocorrência;

6. Encaminhar aos serviços especializados (Médico, Psicólogo e Assistente Social).

Fonte: Criado pelos autores (2021).

As vantagens na construção de um POP para nortear a prática clínica do enfermeiro é necessário que haja um direcionamento na tomada de decisões na prática assistencial, bem como trazer melhorias na qualidade do atendimento do profissional de enfermagem afim de proporcionar uma ação educativa frente as suas possíveis fragilidades e potencialidades no cuidado prestado ao paciente ${ }^{(13)}$. Para objetivar a tomada de decisão dos enfermeiros no atendimento a criança vítima de violência, foi construído um Fluxograma contendo as seguintes variáveis: I) Consulta de Enfermagem; II) Escuta Terapêutica; III) Anamnese; IV) Exame Físico; V) Notificação Compulsória e VI) Encaminhamento aos Serviços Especializados.

Quanto a Consulta de Enfermagem, a Resolução no 568/2018 do Conselho Federal de Enfermagem (COFEN) ${ }^{(14)}$ estabelece este como ato privativo do enfermeiro, na qual o profissional irá identificar problemas de saúde, bem como prescrever e implementar medidas com a finalidade de prestar uma assistência integral, humanizada e qualificada. No que tange a Escuta Terapêutica, esta modalidade de atendimento terapêutico/assistencial promovida por diferentes profissionais da saúde, dentre eles o enfermeiro que por meio da escuta qualificada, irá estabelecer vínculo com o paciente, sendo um instrumento importante para a obtenção de informações, como uma habilidade essencial para o enfermeiro nos cuidados prestados ${ }^{(15)}$.

A Anamnese tem o intuito de criar uma boa relação interpessoal entre a equipe, a criança e pais e/ou responsáveis, visando compreender as informações necessárias por meio do detalhamento da consulta de enfermagem. Além disso, é importante investigar a queixa principal, sinais e sintomas, o responsável legal, o ambiente familiar e os cuidados peculiares ao crescimento e desenvolvimento da criança ${ }^{(16)}$. 
O enfermeiro deve receber a criança, pais e/ou responsáveis com atenção, permeando as seguintes condutas: sentar-se de frente a criança, pais e/ou responsáveis sem nenhuma barreira; avaliar o estado de saúde, emocional e comportamental da criança de acordo com a sua faixa etária; estabelecer as necessidades da criança, respeitando seus limites e queixa principal; chamar a criança pelo nome e realizar perguntas abertas; esclarecer as etapas do atendimento de enfermagem a criança, pais e/ou responsáveis e adotar uma postura ética, sigilosa e empática na consulta de enfermagem ${ }^{(17)}$.

No que compreende ao Exame Físico, este é fundamental para detecção de sinais clínicos da violência, em que este profissional deverá colher seguintes dados no prontuário: peso, altura, sinais vitais, estado clínico, situação vacinal e identificar os principais tipos e locais de lesões nas regiões, como por exemplo, cabeça e pescoço, mama, tóracoabdominal, membros superiores, inferiores e genitais ou extragenitais ${ }^{(18-19-20)}$.

Em relação a casos suspeitos e/ou confirmados de violência, o enfermeiro deverá utilizar a ficha de Notificação Compulsória (SINAN) para fins de investigação criminal, o qual os casos de violência contra criança deverão ser, obrigatoriamente, comunicados ao Conselho Tutelar da respectiva cidade, sem deixar de comunicar os outros órgãos competentes, como as autoridades policiais que acionaram imediatamente o Ministério Público, conforme a Lei $\mathrm{n}^{\mathrm{o}} 13.431$, de 4 de abril de 2017 como o registro do Boletim de Ocorrência ${ }^{(3-18-21)}$.

É relevante que o enfermeiro encaminhe a vítima para os serviços especializados, sendo eles, o Médico, o Assistente Social e o Psicólogo. A atuação médica em casos de violência contra a criança deve ser realizada pelo pediatra, clínico e/ou ginecologista, que posteriormente ao exame físico solicitará possíveis exames como sorologias, culturas em geral, e outros exames sanguíneos complementares se necessário, bem como a prescrição de medicamentos e/ou quimioprofilaxia ${ }^{(20-22)}$.

O Atendimento Psicológico as crianças em situações de violência irão proporcionar uma experiência terapêutica, a fim de minimizar os efeitos e impactos causados pela violência, levando a intervenções como avaliação psicológica, psicoterapia individual e em grupo em diferentes fases do atendimento ${ }^{(20-23)}$.

Durante o atendimento do Serviço Social a criança em situações de violência ocorre intervenção não apenas a vítima, podendo esta ser estendida aos familiares e/ou responsáveis legais, com intuito de promover apoio psicossocial ${ }^{(20-24)}$.

Na terceira etapa sobre avaliação das barreiras para o uso do conhecimento, foi evidenciado pelo enfermeiro: ausência de habilidades para desenvolver um atendimento 
sistematizado; insegurança e/ou resistência em realizar mudanças nas práticas profissionais com base nas evidências científicas e falta de apoio ou incentivo entre os profissionais das equipes da ESF. Em vista disso, a falta de conscientização em aderir às mudanças tem impacto direto na implementação de melhorias e segurança da criança vítima de violência, sendo necessário envolver o profissional no planejamento e execução de estratégias que visem a promoção e prevenção de saúde ${ }^{(25)}$.

No referente a quarta etapa sobre seleção, adaptação, implementação e intervenção, foram apontados os seguintes determinantes: melhorias na prática clínica, como facilitador na tomada de decisão para intervir de maneira crítica e sistematizada ao atendimento da criança vítima de violência, bem como o encaminhamento a serviços especializados, promovendo práticas profissionais em consonância com as evidencias científicas, permitindo assim a participação e contribuição do enfermeiro na assistência prestada ${ }^{(26)}$.

Condizente a quinta etapa sobre o monitoramento do uso do conhecimento, sugere-se a aplicação de um teste antes e após se empregar o uso deste POP para avaliar o grau de conhecimento dos enfermeiros, sendo indispensável sua atualização com a finalidade de buscar normas e diretrizes atualizadas para capacitar os enfermeiros, a fim de que possam identificar e conduzir sua tomada de decisão frente aos casos de violência infantil ${ }^{(18)}$.

$\mathrm{Na}$ sexta etapa sobre avaliação dos resultados, é importante que os profissionais realizem treinamentos com intuito de identificar as principais dificuldades sobre a temática, participando de grupos focais para que os mesmos exponham as suas ideias, recomendem e/ou desenvolvam pesquisas científicas sobre a violência infantil de acordo com o trabalho do enfermeiro da $\operatorname{ESF}^{\text {(18-27). }}$

Por fim, a sétima etapa que esta relacionada ao uso sustentável do conhecimento, infere ser imprescindível que os enfermeiros da ESF realizem a atualização do POP e a capacitação dos profissionais, antecipando esse treinamento mediante mudanças recentes de normas e regulamentos. Além disso, é fundamental a realização de discussões para melhoria das ações por meio de políticas, investimentos e novas diretrizes em parcerias com organizações de níveis superiores ${ }^{(13)}$.

Mediante ao exposto, justifica-se a inserção no serviço de ESF um Fluxograma de atendimento voltado a assistência de enfermagem a criança vítima de violência, como visto na figura 1 abaixo, apontado como proposta dos autores para conduzir a tomada de decisão durante o atendimento. 
Figura 1 - Fluxograma de atendimento voltado a assistência de enfermagem a criança vítima de violência.

\section{FLUXOGRAMA DE ATENDIMENTO VOLTADO A ASSISTÊNCIA DE ENFERMAGEM A CRIANÇA VÍTIMA} DE VIOLÊNCIA

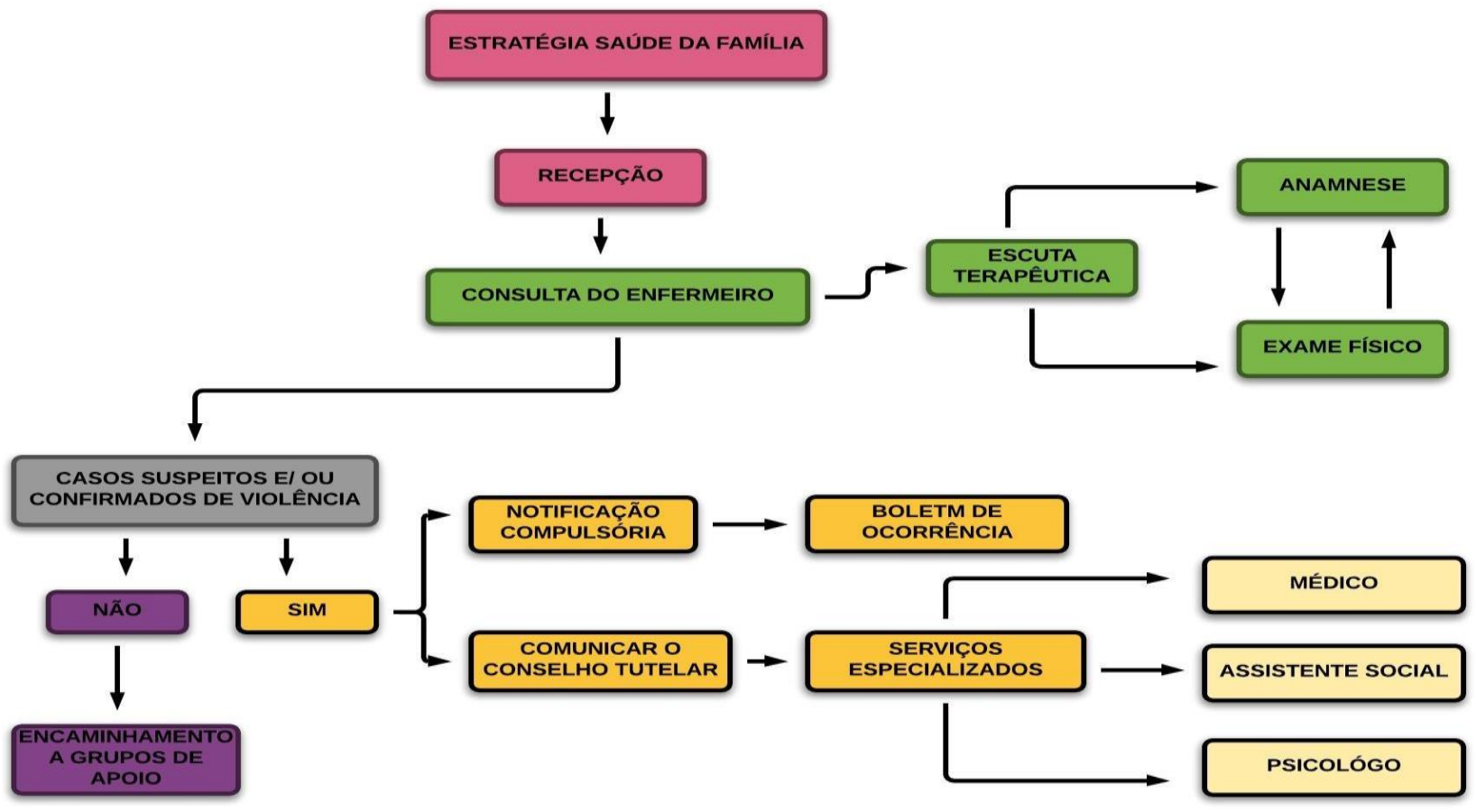

Fonte: Os autores

\section{CONCLUSÃO}

O estudo desenvolveu um POP com o intuito de padronizar a ação referente ao atendimento de enfermeiros da ESF no cuidado a criança vítima de violência, como proposta de inclusão nos serviços de ESF para auxiliar os profissionais nos caminhos que irão conduzir em um atendimento qualificado e especializado.

A elaboração desse POP irá contribuir para a capacitação dos profissionais e sua implementação no serviço da ESF para que o enfermeiro possa treinar sua equipe e outros enfermeiros, bem como sistematizar uma assistência com maior participação de outros profissionais da saúde.

A limitação encontrada foi a ausência de estudos clínicos que tivesse uma proposta de implantar um POP no serviço de ESF. Portanto, sugere-se novos estudos que construam protocolos no atendimento a mulheres, homens e idosos vítimas de violência não somente na ESF, mas também nos serviços hospitalares.

\section{REFERÊNCIAS}

1. Krug EG, Dahlberg LL, Mercy JA, Zwi $\mathrm{AB}$, Lozano $\mathrm{R}$, organizadores. World report 
on violence and health. Geneva $(\mathrm{CH})$ : World Health Organization, 2002.

2. Cerqueira D, Bueno S, Alves PP, Lima RS, Ferreira $\mathrm{H}$ et al. Atlas da Violência $2020-$ Ipea e FBSP. [internet]. Distrito Federal: Instituto de Pesquisa Econômica Aplicada. 2020. 96p. [acesso em 11 de mai de 2021]. Disponível em: https://www.ipea.gov.br/atlasviolencia/downl oad/24/atlas-da-violencia-2020

3. Cerqueira D, Bueno S, Alves PP, Lima RS, Ferreira $\mathrm{H}$ et al. Atlas da Violência $2020-$ Ipea e FBSP. [internet]. Distrito Federal: Instituto de Pesquisa Econômica Aplicada. 2020. 96p. [acesso em 11 de mai de 2021]. Disponível em: https://www.ipea.gov.br/atlasviolencia/downl oad/24/atlas-da-violencia-2020

4. Lei $\mathrm{n}^{\mathrm{o}} 12.845$, de $1^{\mathrm{o}}$ de agosto de 2013 (BR). Lei do minuto seguinte. [internet] Brasília: DF, 2013. [acesso 06 mai 2021]. Disponível em: https://leidominutoseguinte.mpf.mp.br

5. Melo RA, Souza SDL, Bezerra CS. Cuidados de enfermagem à criança e adolescente em violência doméstica na visão de graduandos de enfermagem. Av en Enfermería. [Internet]. 2017; [citado 2 de set de 2021]; 35(3): 293-302 Disponível em: http://www.scielo.org.co/scielo.php?script=sc i_arttext\&pid=S0121-45002017000300293

6.Egry EY. et al. ApostólicoI MR, MoraisII TCP, LisboaII CCR. Enfrentar a violência infantil na Atenção Básica: como os profissionais percebem? Rev. Bras. Enferm. [Internet]. 2017; [citado 2 de set de 2021]; 70 (1): 119-25 10. Disponível em: https://doi.org/10.1590/0034-7167-2016-0009

7. Polit DF, Beck CT. Fundamentos de pesquisa em enfermagem: avaliação de evidências para a prática da enfermagem. 7a ed. Porto Alegre: Artmed; 2011.

8 Graham ID, Logan J, Harrison MB, Straus SE, Tetroe $J$ et al. Lost in knowledge translation: time for a map? J Contin Educ Health Prof. [Internet]. 2006. [citado 2 de set de 2021]; 26(1):13 24. Disponível em em: https://doi.org/10.1002/chp.47

9. Vieira ACG, Gastaldo D, Harrison D. Como traduzir o conhecimento científico à prática? Conceitos, modelos e aplicação. Rev Bras Enferm. [Internet]. 2020; [citado 2 de set de 2021]; 73. Disponível em: https://doi.org/10.1590/0034-7167-2019-0179

10. Crossetti MGO, Goes MGO. Translação do conhecimento: um desafio para prática de enfermagem. Rev. Gaúcha Enferm. [Internet]. 2017; [citado 2 de set de 2021]; 38(2). Disponível em: https://doi.org/10.1590/19831447.2017.02.74266

11.Mendes KDS, Silveira RCCP, Galvão CM. Método de pesquisa para a incorporação de evidências na saúde e na enfermagem. Texto Contexto Enferm [Internet].2008; [citado 2 de set de 2021]; 17 (4): 758-64 Disponível em: https://doi.org/10.1590/S0104$\underline{07072008000400018}$

12. Amorim de Ávila J, Netto de Oliveira AM. Conhecimento dos Enfermeiros frente ao abuso sexual. Av. enferm. [Internet]. 1 de julio de 2012 [citado 2 de set de 2021];30(2):47-55. Disponível em: https://revistas.unal.edu.co/index.php/avenfer $\mathrm{m} /$ article/view/36161

13. Sales CB, Bernardes A, Gabriel CS, Brito MFP, Moura AA et al. Standard Operational Protocols in professional nursing practice: use, weaknesses and potentialities. Rev Bras Enferm. [Internet]. 2018; 71(1):126-34 Disponível em: https://doi.org/10.1590/0034$\underline{\text { 7167-2016-0621 }}$

14. Conselho Federal de Enfermagem (Cofen). Resolução COFEN no 568/2018, de 20 de fevereiro de 2018. Regulamenta o funcionamento dos consultórios e clínicas de enfermagem. Brasília: DF, 2018. [acesso 06 jul 2021]. Disponível em: http://www.cofen.gov.br/resolucao-cofen-no$\underline{0568-2018 \text { 60473.html }}$

15. Mesquita AC, Carvalho ECA. A Escuta Terapêutica como estratégia de intervenção em saúde: uma revisão integrativa. Rev Esc Enferm USP. [Internet]. 2014. [citado 2 de set 
de 2021];48(6); 1227-1236. [acesso 06 jul 2021]. Disponível em: https://www.scielo.br/pdf/reeusp/v48n6/pt_00 80-6234-reeusp-48-06-1127.pdf

16. Ferreira AL. A criança vítima de violência. Revista de Pediatria SOPERJ. 2012;13(2):4-9. [acesso 06 jul 2021]. Disponível em: http://revistadepediatriasoperj.org.br/detalhe_ artigo.asp?id=612

17. Soares VFR, Dantas DV, Dantas RAN, Costa IKFC, Leite JEL. Atuação do enfermeiro no atendimento à criança vítima de trauma: revisão de literatura. Carpe Diem: Revista Cultural e Científica do UNIFACEX. [Internet]. 2015; [citado 2 de set de 2021]; 13(11). Disponível em: https://periodicos.unifacex.com.br/Revista/arti cle/view/643/pdf

18. Silva SA, Ceribelli C. O papel do enfermeiro frente a violência infantil na atenção primária. Revista Eletrônica Acervo Enfermagem. [Internet]. 2021; [citado 2 de set de 2021]; 8 (e5001): Disponível em: https://doi.org/10.25248/reaenf.e5001.2021

19. Ministério da Saúde (Br). Secretaria de políticas para as mulheres. Norma Técnica. Atenção humanizada às pessoas em situação de violência sexual com registro de informações e coleta de vestígios. Brasília: DF, 2015. [acesso 06 jul 2021]. Disponível em:

https://bvsms.saude.gov.br/bvs/publicacoes/at encao_humanizada_pessoas_violencia_sexual norma_tecnica.pdf

20. Ministério da Saúde (Br). Secretaria de Estado de Saúde do Distrito Federal. Manual para atendimento às vítimas de violência na rede de saúde pública do DF. [internet] Brasília: DF, 2009. [acesso 06 jul 2021]. Disponível em: http://bvsms.saude.gov.br/bvs/publicacoes/ma nual_atendimento_vitimas_violencia_saude_p ublica_DF.pdf

21. Ministério da Saúde (Br). Secretaria de Vigilância em Saúde. Ficha de notificação/ investigação individual violência doméstica, sexual e/ou outras violências interpessoais. Brasília, DF: Ministério da Saúde; 2006. [acesso 06 jul 2021]. Disponível em: https://bvsms.saude.gov.br/bvs/folder/ficha_n otificacao_violencia_domestica.pdf

22. Menezes MLB, Araújo MAL, Santos ASD, Gir El, Bermúdez XPD. Protocolo Brasileiro para Infecções Sexualmente Transmissíveis 2020: violência sexual. Epidemiol. Serv. Saúde [online]. 2021, vol.30, n.esp1, e2020600. Disponível em. http://dx.doi.org/10.1590/s16794974202100018.esp1

23. Bezerra KP, Monteiro AI. Violência Intrafamiliar contra criança: intervenção de enfermeiros da estratégia de saúde da família. Revista Rene. [Internet]. 2012; [citado 2 de set de 2021];13(2); 354-64. Disponível em: http://periodicos.ufc.br/rene/article/view/3926

24. Seger ACBP, Caldart P, Grossi PK. Desvelando a violência contra as crianças em um hospital universitário: desafios para o Serviço Social. Textos \& Contextos. [Internet]. 2010; [citado 2 de set de 2021]; 9(1):118-13. Disponível em: https://www.redalyc.org/articulo.oa?id=32152 7166012

25. Reis GAX, Oliveira JLC, Ferreira AMD, Vituri DW, Marcon SS et al. Dificuldades para implantar estratégias de segurança do paciente: perspectivas de enfermeiros gestores. Revista Gaúcha de Enfermagem [Internet]. 2019; [citado 2 de set de 2021]; v. 40(spe). Disponível em: https://doi.org/10.1590/1983$\underline{1447.2019 .20180366}$

26._Sommer D, Franciscatto LG, Getelina CO, Salvador K. Caracterização da violência contra crianças e adolescentes: indicativos para a prática do enfermeiro. Revista de Enfermagem. [Internet].2017; [citado 2 de set de 2021]; 13(13):14-28. Disponível em: http://revistas.fw.uri.br/index.php/revistadeen fermagem/article/view/2607/2560

27. Carlos, DM, Padua, EMM, Fernandes MID, Leitão MNC, Ferriani MGC. Rev Gaúcha Enferm. [internet] 2016; [citado 2 de 
set de 2021]; 37(esp):e72859 Disponível em: https://doi.org/10.1590/1983-

$\underline{1447.2016 . e s p .72859}$

\section{Autor correspondente}

Jhuliano Silva Ramos de Souza, Rua da Liberdade, n 72, Vila Betânia, CEP: 37137090, Alfenas, Minas Gerais, Brasil Cel: +55(35) 988667794

Submissão: 2021-09-03

Aprovado: 2021-10-26 